\title{
Sums of products of Apostol-Bernoulli and Apostol-Euler polynomials
}

Yuan $\mathrm{He}^{1 *}$ and Serkan Araci ${ }^{2}$

${ }^{*}$ Correspondence: hyyhe@aliyun.com ${ }^{1}$ Faculty of Science, Kunming University of Science and Technology, Kunming, Yunnan 650500, People's Republic of China Full list of author information is available at the end of the article

\begin{abstract}
In this paper, a further investigation for the Apostol-Bernoulli and Apostol-Euler polynomials and numbers is performed. Some closed formulae of sums of products of any number of Apostol-Bernoulli and Apostol-Euler polynomials and numbers are established by applying the generating function methods and some summation transform techniques. It turns out that some well-known results are derived as special cases.

MSC: $11 \mathrm{~B} 68 ; 05 \mathrm{~A} 19$

Keywords: Bernoulli polynomials and numbers; Euler polynomials and numbers; Apostol-Bernoulli polynomials and numbers; Apostol-Euler polynomials and numbers; summation formulae
\end{abstract}

\section{Introduction}

The classical Bernoulli polynomials $B_{n}(x)$ and Euler polynomials $E_{n}(x)$ are usually defined by means of the following generating functions:

$$
\frac{t e^{x t}}{e^{t}-1}=\sum_{n=0}^{\infty} B_{n}(x) \frac{t^{n}}{n !} \quad(|t|<2 \pi) \quad \text { and } \quad \frac{2 e^{x t}}{e^{t}+1}=\sum_{n=0}^{\infty} E_{n}(x) \frac{t^{n}}{n !} \quad(|t|<\pi) .
$$

In particular, the rational numbers $B_{n}=B_{n}(0)$ and integers $E_{n}=2^{n} E_{n}(1 / 2)$ are called the classical Bernoulli numbers and Euler numbers, respectively.

As is well known, the classical Bernoulli and Euler polynomials and numbers play important roles in different areas of mathematics such as number theory, combinatorics, special functions and analysis. Numerous interesting properties for them can be found in many books; see, for example, [1-4]. Here is the well-known Euler formula on the classical Bernoulli numbers:

$$
\sum_{k=0}^{n}\left(\begin{array}{l}
n \\
k
\end{array}\right) B_{k} B_{n-k}=-n B_{n-1}-(n-1) B_{n} \quad(n \geq 1)
$$

This formula has been extended in different directions by many authors. For example, a direct generalisation of (1.2) is the following identity on the classical Bernoulli polynomials due to Nörlund [5]:

$$
\sum_{k=0}^{n}\left(\begin{array}{l}
n \\
k
\end{array}\right) B_{k}(x) B_{n-k}(y)=-n(x+y-1) B_{n-1}(x+y)-(n-1) B_{n}(x+y) \quad(n \geq 1),
$$


which has been generalised to a symmetric form by He and Zhang [6]. We also mention [7-11] for further discoveries of the above Nörlund result following the work of Carlitz [12], Agoh and Dilcher [13, 14]. On the other hand, Eie [15], Sitaramachandraro and Davis [16] generalised (1.2) to sums of products of three and four Bernoulli numbers. After that, Sankaranarayanan [17] gave the closed expression of sums of products of five Bernoulli numbers, and Zhang [18] derived the closed ones of sums of products of less than and equal to seven Bernoulli numbers. In particular, Dilcher [19] obtained some closed formulae of sums of products of any number of classical Bernoulli and Euler polynomials and numbers. Petojević and Srivastava [20-22] got several new formulae of sums of products of any number of classical Bernoulli numbers including the Euler type and Dilcher type sums of products of Bernoulli numbers. Recently, Kim [23] developed a new approach to give the closed formula of sums of products of any number of classical Bernoulli numbers by using the relation of values at non-positive integers of the important representation of the multiple Hurwitz zeta function in terms of the Hurwitz zeta function. Further, Kim and $\mathrm{Hu}$ [24] obtained the closed formula of sums of products of any number of ApostolBernoulli numbers by expressing the sums of products of the Apostol-Bernoulli polynomials in terms of the special values of multiple Hurwitz-Lerch zeta functions at non-positive integers.

We now turn to the Apostol-Bernoulli polynomials $\mathcal{B}_{n}(x ; \lambda)$ and Apostol-Euler polynomials $\mathcal{E}_{n}(x ; \lambda)$, which are usually defined by means of the following generating functions (see, e.g., $[25,26])$ :

$$
\frac{t e^{x t}}{\lambda e^{t}-1}=\sum_{n=0}^{\infty} \mathcal{B}_{n}(x ; \lambda) \frac{t^{n}}{n !} \quad(|t|<2 \pi \text { if } \lambda=1 ;|t|<|\log \lambda| \text { otherwise })
$$

and

$$
\frac{2 e^{x t}}{\lambda e^{t}+1}=\sum_{n=0}^{\infty} \mathcal{E}_{n}(x ; \lambda) \frac{t^{n}}{n !} \quad(|t|<\pi \text { if } \lambda=1 ;|t|<|\log (-\lambda)| \text { otherwise }) .
$$

Moreover, $\mathcal{B}_{n}(\lambda)=\mathcal{B}_{n}(0 ; \lambda)$ and $\mathcal{E}_{n}(\lambda)=2^{n} \mathcal{E}_{n}(1 / 2 ; \lambda)$ are called the Apostol-Bernoulli numbers and Apostol-Euler numbers, respectively. Obviously $\mathcal{B}_{n}(x ; \lambda)$ and $\mathcal{E}_{n}(x ; \lambda)$ reduce to $B_{n}(x)$ and $E_{n}(x)$ when $\lambda=1$. It is worth noticing that the Apostol-Bernoulli polynomials were firstly introduced by Apostol [27] (see also Srivastava [28] for a systematic study) in order to evaluate the value of the Hurwitz-Lerch zeta function. For some elegant results and nice methods on these polynomials and numbers, one is referred to [29-38].

In the present paper, we will be concerned with some closed formulae of sums of products of any number of Apostol-Bernoulli and Apostol-Euler polynomials and numbers. The idea stems from the two identities of Xu and Cen [39] applying the famous Faà di Bruno formula to answer a problem posed by Guo and Qi [40]. We prove the two results due to $\mathrm{Xu}$ and Cen in a brief way again. As further applications, we obtain some closed formulae of sums of products of any number of Apostol-Bernoulli and Apostol-Euler polynomials and numbers by applying the generating function methods and some summation transform techniques. It turns out that some known results including the ones stated in $[19,24]$ are derived as special cases. 


\section{Some auxiliary results}

We firstly recall the Stirling numbers of the first kind $s(n, k)$ and the Stirling numbers of the second kind $S(n, k)$ which can be found in the standard book [41]. The Stirling numbers of the first kind are the coefficients in the expansion

$$
(x)_{n}=\sum_{k=0}^{n} s(n, k) x^{k} \quad(n \geq 0)
$$

and the Stirling numbers of the second kind are characterised by the identity

$$
x^{n}=\sum_{k=0}^{n} S(n, k)(x)_{k} \quad(n \geq 0) .
$$

The notation $(x)_{n}$ appearing in (2.1) and (2.2) stands for the falling factorial $(x)_{n}$ of order $n$ defined by $(x)_{0}=1$ and $(x)_{n}=x(x-1)(x-2) \cdots(x-n+1)$ for positive integer $n$ and complex number $x$. In fact, the Stirling numbers of the first kind $s(n, k)$ and the Stirling numbers of the second kind $S(n, k)$ can be defined by means of the following generating functions:

$$
\frac{(\ln (1+t))^{k}}{k !}=\sum_{n=k}^{\infty} s(n, k) \frac{t^{n}}{n !} \text { and } \quad \frac{\left(e^{t}-1\right)^{k}}{k !}=\sum_{n=k}^{\infty} S(n, k) \frac{t^{n}}{n !}
$$

If making use of (2.3) then one can easily obtain the pair of classical inverse relations (see, e.g., [41, p.144]):

$$
f(n)=\sum_{k=0}^{n} s(n, k) g(k) \Longleftrightarrow g(n)=\sum_{k=0}^{n} S(n, k) f(k),
$$

with $n$ being non-negative integer and $f(n)$ and $g(n)$ being two sequences.

Motivated by two identities appearing in [42], Guo and Qi [40, 43] posed the following problem: for $t \neq 0$ and positive integer $n$, determine the numbers $a_{n, k-1}$ for $1 \leq k \leq n$ such that

$$
\frac{1}{\left(1-e^{-t}\right)^{n}}=1+\sum_{k=1}^{n} a_{n, k-1}\left(\frac{1}{e^{t}-1}\right)^{(k-1)} .
$$

Stimulated by this problem, the authors [40] made use of the mathematical induction to establish eight identities which reveal the functions $1 /\left(1-e^{ \pm t}\right)^{n}$ and the derivatives $\left(1 /\left(e^{ \pm t}-\right.\right.$ $1))^{(k)}$ can be expressed by each other by linear combinations with coefficients involving the combinatorial numbers and Stirling numbers of the second kind. Further, $\mathrm{Xu}$ and Cen [39] applied the famous Faà di Bruno formula to unify the eight identities due to Guo and Qi [40] to two identities involving two parameters $\alpha$ and $\lambda$. We next state their results and give a brief proof.

Theorem 2.1 ([39]) Let $n$ be a non-negative integer and $\alpha, \lambda$ two parameters. Then

$$
\left(\frac{1}{1-\lambda e^{\alpha t}}\right)^{(n)}=\sum_{k=1}^{n+1} \frac{(-1)^{n+k-1} \alpha^{n}(k-1) ! S(n+1, k)}{\left(1-\lambda e^{\alpha t}\right)^{k}},
$$


and for positive integer $n$,

$$
\frac{1}{\left(1-\lambda e^{\alpha t}\right)^{n}}=\sum_{k=1}^{n} \frac{(-1)^{n-k} s(n, k)}{(n-1) ! \alpha^{k-1}}\left(\frac{1}{1-\lambda e^{\alpha t}}\right)^{(k-1)} .
$$

Proof In view of the binomial series

$$
(1+t)^{\beta}=\sum_{n=0}^{\infty}\left(\begin{array}{l}
\beta \\
n
\end{array}\right) t^{n} \quad(\beta \text { complex number })
$$

we discover

$$
\frac{1}{\left(1-\lambda e^{\alpha t}\right)^{n}}=\sum_{j=0}^{\infty}\left(\begin{array}{c}
-n \\
j
\end{array}\right)\left(-\lambda e^{\alpha t}\right)^{j}=\sum_{j=0}^{\infty}\left(\begin{array}{c}
n+j-1 \\
j
\end{array}\right)\left(\lambda e^{\alpha t}\right)^{j} .
$$

If taking $x=-j$ in (2.1) then we obtain, for positive integer $n$,

$$
\frac{1}{(n-1) !} \sum_{k=1}^{n} s(n, k)(-1)^{n-k} j^{k-1}=\left(\begin{array}{c}
n+j-1 \\
j
\end{array}\right) .
$$

Applying (2.10) to (2.9) yields

$$
\frac{1}{\left(1-\lambda e^{\alpha t}\right)^{n}}=\frac{1}{(n-1) !} \sum_{k=1}^{n}(-1)^{n-k} s(n, k) \sum_{j=0}^{\infty}\left(\lambda e^{\alpha t}\right)^{j} j^{k-1} .
$$

It is easy to see from (2.9) that

$$
\left(\frac{1}{1-\lambda e^{\alpha t}}\right)^{(k-1)}=\sum_{j=0}^{\infty} \lambda^{j}\left(e^{\alpha t j}\right)^{(k-1)}=\alpha^{k-1} \sum_{j=0}^{\infty}\left(\lambda e^{\alpha t}\right)^{j} j^{k-1} .
$$

Thus, combining (2.11) and (2.12) gives the formula (2.7). It follows from (2.4) and (2.7) that (2.6) is complete. This concludes the proof.

It becomes obvious that in the case $\alpha=1$ in (2.7) arises, for positive integer $n$,

$$
\frac{1}{\left(\lambda e^{t}-1\right)^{n}}=\sum_{k=1}^{n} \frac{(-1)^{k-1} s(n, k)}{(n-1) !}\left(\frac{1}{\lambda e^{t}-1}\right)^{(k-1)}
$$

and

$$
\frac{1}{\left(\lambda e^{t}+1\right)^{n}}=\sum_{k=1}^{n} \frac{(-1)^{n-k} s(n, k)}{(n-1) !}\left(\frac{1}{\lambda e^{t}+1}\right)^{(k-1)} .
$$

We next give another auxiliary result as follows.

Theorem 2.2 Let $n$ be a non-negative integer. Then

$$
e^{x t}(f(y, t))^{(n)}=\sum_{m=0}^{\infty}\left[\sum_{k=0}^{n}\left(\begin{array}{l}
n \\
k
\end{array}\right)(-x)^{n-k} f_{m+k}(x+y)\right] \frac{t^{m}}{m !},
$$


and for positive integer $r$,

$$
\begin{aligned}
e^{x t}(g(y, t))^{(n)}= & \sum_{m=0}^{\infty}\left[\sum_{k=0}^{n}\left(\begin{array}{l}
n \\
k
\end{array}\right)(-x)^{n-k} \frac{f_{m+k+r}(x+y)}{\langle m+k+1\rangle_{r}}\right. \\
& \left.+\frac{(-1)^{n+1} x^{m+n+1}}{(r-1) !} \int_{0}^{1} t^{m}(1-t)^{n} f_{r-1}(x+y-x t) d t\right] \frac{t^{m}}{m !},
\end{aligned}
$$

where $\langle x\rangle_{n}$ denotes the rising factorial $\langle x\rangle_{n}$ of order $n$ defined by $\langle x\rangle_{0}=1$ and $\langle x\rangle_{n}=$ $x(x+1)(x+2) \cdots(x+n-1)$ for positive integer $n$ and complex number $x, f(y, t)$ is denoted by $f(y, t)=\sum_{m=0}^{\infty} f_{m}(y) \frac{t^{m}}{m !}, g(y, t)$ is denoted by $g(y, t)=\sum_{m=0}^{\infty} \frac{f_{m+r}(y)}{\langle m+1\rangle_{r}} \cdot \frac{t^{m}}{m !}$ and $\left\{f_{n}(x)\right\}_{n=0}^{\infty}$ is a sequence of polynomials given by

$$
\sum_{n=0}^{\infty} f_{n}(x) \frac{t^{n}}{n !}=F(t) e^{(x-1 / 2) t}
$$

with $F(t)$ being a formal power series.

Proof See [44, (2.6), (3.11)] for details.

There follow some special cases of Theorem 2.2. By setting $y=0$ and $f(y, t)=2 /\left(\lambda e^{t}+1\right)$ in (2.15), we obtain, for non-negative integers $m, n$,

$$
\left[\frac{t^{m}}{m !}\right]\left(e^{x t}\left(\frac{2}{\lambda e^{t}+1}\right)^{(n)}\right)=\sum_{k=0}^{n}\left(\begin{array}{l}
n \\
k
\end{array}\right)(-x)^{n-k} \mathcal{E}_{m+k}(x ; \lambda),
$$

where $\left[t^{n}\right] f(t)$ stands for the coefficients of $\left[t^{n}\right]$ in $f(t)$. On the other hand, since $\mathcal{B}_{0}(x ; \lambda)=1$ when $\lambda=1$ and $\mathcal{B}_{0}(x ; \lambda)=0$ when $\lambda \neq 1$ (see, e.g., [25]), so by setting $\mathcal{B}_{0}(x ; \lambda)=\delta_{1, \lambda}$ we get

$$
\frac{1}{\lambda e^{t}-1}-\frac{\delta_{1, \lambda}}{t}=\sum_{m=0}^{\infty} \frac{\mathcal{B}_{m+1}(\lambda)}{m+1} \cdot \frac{t^{m}}{m !}
$$

It is easy to see from the properties of the Beta and Gamma functions that, for nonnegative integers $m, n$,

$$
\int_{0}^{1} t^{m}(1-t)^{n} d t=\frac{m ! \cdot n !}{(m+n+1) !}
$$

Thus, by setting $r=1, y=0$ and $g(y, t)=1 /\left(\lambda e^{t}-1\right)-\delta_{1, \lambda} / t$ in (2.16), with the help of (2.19) and (2.20), we obtain, for non-negative integers $m, n$,

$$
\left[\frac{t^{m}}{m !}\right]\left(e^{x t}\left(\frac{1}{\lambda e^{t}-1}\right)^{(n)}\right)=\sum_{k=0}^{n}\left(\begin{array}{l}
n \\
k
\end{array}\right)(-x)^{n-k} \frac{\mathcal{B}_{m+k+1}(x ; \lambda)}{m+k+1} .
$$

We shall make use of the above formulae (2.13), (2.14), (2.18) and (2.21) to give some closed formulae of sums of products of any number of Apostol-Bernoulli and Apostol-Euler polynomials and numbers in the next section. 


\section{The restatement of main results}

Before stating our main results, we begin by introducing the Stirling cycle numbers $\left[\begin{array}{l}n \\ k\end{array}\right]$ given by the relation

$$
x(x+1) \cdots(x+n-1)=\sum_{k=0}^{n}\left[\begin{array}{l}
n \\
k
\end{array}\right] x^{k} .
$$

It is obvious from (2.1) that $s(n, k)=(-1)^{n-k}\left[\begin{array}{l}n \\ k\end{array}\right]$. In fact, the Stirling cycle numbers can be defined recursively by (see, e.g., $[4,29])$

$$
\begin{aligned}
& {\left[\begin{array}{l}
0 \\
0
\end{array}\right]=1, \quad\left[\begin{array}{l}
n \\
0
\end{array}\right]=\left[\begin{array}{l}
0 \\
n
\end{array}\right]=0 \quad \text { and }} \\
& {\left[\begin{array}{c}
n+1 \\
k
\end{array}\right]=n\left[\begin{array}{l}
n \\
k
\end{array}\right]+\left[\begin{array}{c}
n \\
k-1
\end{array}\right] \quad(n, k \geq 1) .}
\end{aligned}
$$

In this section we always denote by $p_{n, m}(x)$ a polynomial given by (see, e.g., $\left.[23,24]\right)$

$$
\begin{aligned}
p_{n, m}(x) & =\frac{1}{(n-1) !} \sum_{k=m}^{n-1}\left(\begin{array}{c}
k \\
m
\end{array}\right)\left[\begin{array}{c}
n \\
k+1
\end{array}\right](-x)^{k-m} \\
& =\frac{(-1)^{n-m-1}}{(n-1) !} \sum_{k=m}^{n-1}\left(\begin{array}{l}
k \\
m
\end{array}\right) s(n, k+1) x^{k-m},
\end{aligned}
$$

and we also denote by $\left(\begin{array}{c}n \\ r_{1}, \ldots, r_{k}\end{array}\right)$ the multinomial coefficient defined by

$$
\left(\begin{array}{c}
n \\
r_{1}, \ldots, r_{k}
\end{array}\right)=\frac{n !}{r_{1} ! \cdots r_{k} !} \quad\left(n, r_{1}, \ldots, r_{k} \geq 0\right) \text {. }
$$

We next explore the closed formulae of sums of products of any number of ApostolBernoulli and Apostol-Euler polynomials and numbers. The discovery depends on the following identity:

$$
\left(\frac{t}{\lambda e^{t}-1}\right)^{r}\left(\frac{2}{\lambda e^{t}+1}\right)^{m-r} e^{\left(x_{1}+x_{2}+\cdots+x_{m}\right) t}=\frac{t^{r} 2^{m-r} e^{y t}}{\left(\lambda e^{t}-1\right)^{r}\left(\lambda e^{t}+1\right)^{m-r}},
$$

where $r$ is a non-negative integer, $m$ is a positive integer with $0 \leq r \leq m$ and $y=x_{1}+x_{2}+$ $\cdots+x_{m}$. On taking $r=m$ in (3.5) by (1.4) we get

$$
\left[\frac{t^{n}}{n !}\right]\left(\frac{t^{m} e^{y t}}{\left(\lambda e^{t}-1\right)^{m}}\right)=\sum_{\substack{k_{1}+\cdots+k_{m}=n \\
k_{1}, \ldots, k_{m} \geq 0}}\left(\begin{array}{c}
n \\
k_{1}, \ldots, k_{m}
\end{array}\right) \mathcal{B}_{k_{1}}\left(x_{1} ; \lambda\right) \cdots \mathcal{B}_{k_{m}}\left(x_{m} ; \lambda\right) .
$$

On the other hand, from (2.13) we discover

$$
\begin{aligned}
& {\left[\begin{array}{l}
t^{n} \\
n !
\end{array}\right]\left(\frac{t^{m} e^{y t}}{\left(\lambda e^{t}-1\right)^{m}}\right)} \\
& \quad=\frac{n !}{(n-m) !} \sum_{k=1}^{m} \frac{(-1)^{k-1} s(m, k)}{(m-1) !}\left[\frac{t^{n-m}}{(n-m) !}\right]\left(e^{y t}\left(\frac{1}{\lambda e^{t}-1}\right)^{(k-1)}\right) .
\end{aligned}
$$


If applying (2.21) to the right-hand side of (3.7) then

$$
\begin{aligned}
& {\left[\frac{t^{n}}{n !}\right]\left(\frac{t^{m} e^{y t}}{\left(\lambda e^{t}-1\right)^{m}}\right)} \\
& =\frac{n !}{(n-m) !} \sum_{k=1}^{m} \frac{(-1)^{k-1} s(m, k)}{(m-1) !} \sum_{j=0}^{k-1}\left(\begin{array}{c}
k-1 \\
j
\end{array}\right)(-y)^{k-1-j} \frac{\mathcal{B}_{n+j+1-m}(y ; \lambda)}{n+j+1-m} \text {. }
\end{aligned}
$$

Changing the order of the summation on the right-hand side of (3.8) gives

$$
\begin{aligned}
{\left[\begin{array}{c}
t^{n} \\
n !
\end{array}\right] } & \left(\frac{t^{m} e^{y t}}{\left(\lambda e^{t}-1\right)^{m}}\right) \\
= & \frac{n !}{(n-m) !} \sum_{j=0}^{m-1}(-1)^{j} \frac{\mathcal{B}_{n+j+1-m}(y ; \lambda)}{n+j+1-m} \sum_{k=j+1}^{m}\left(\begin{array}{c}
k-1 \\
j
\end{array}\right) \frac{s(m, k) y^{k-1-j}}{(m-1) !} \\
= & \frac{(-1)^{m-1} n !}{(n-m) !} \sum_{j=0}^{m-1}(-1)^{j} \frac{\mathcal{B}_{n-j}(y ; \lambda)}{n-j} \sum_{k=m-j}^{m}\left(\begin{array}{c}
k-1 \\
m-j-1
\end{array}\right) \frac{s(m, k) y^{k-m+j}}{(m-1) !} \\
= & \frac{(-1)^{m-1} n !}{(n-m) !} \sum_{j=0}^{m-1}(-1)^{j} \frac{\mathcal{B}_{n-j}(y ; \lambda)}{n-j} \\
& \times \frac{1}{(m-1) !} \sum_{k=m-j-1}^{m-1}\left(\begin{array}{c}
k \\
m-j-1
\end{array}\right) s(m, k+1) y^{k-(m-j-1)} .
\end{aligned}
$$

Thus, by equating (3.6) and (3.9) and then applying (3.3) the following result arises.

Theorem 3.1 Let $m$ be a positive integer and $y=x_{1}+x_{2}+\cdots+x_{m}$. Then, for positive integer $n \geq m$,

$$
\begin{aligned}
& \sum_{\substack{k_{1}+\ldots+k_{m}=n \\
k_{1}, \ldots, k_{m} \geq 0}}\left(\begin{array}{c}
n \\
k_{1}, \ldots, k_{m}
\end{array}\right) \mathcal{B}_{k_{1}}\left(x_{1} ; \lambda\right) \cdots \mathcal{B}_{k_{m}}\left(x_{m} ; \lambda\right) \\
& =\frac{(-1)^{m-1} n !}{(n-m) !} \sum_{j=0}^{m-1} p_{m, m-j-1}(y) \frac{\mathcal{B}_{n-j}(y ; \lambda)}{n-j} .
\end{aligned}
$$

There follow some special cases of Theorem 3.1. Obviously the case $\lambda=1$ in Theorem 3.1 is an equivalent version of the classical formula of Dilcher [19, Theorem 3]:

$$
\begin{aligned}
& \sum_{\substack{k_{1}+\ldots+k_{m}=n \\
k_{1}, \ldots, k_{m} \geq 0}}\left(\begin{array}{c}
n \\
k_{1}, \ldots, k_{m}
\end{array}\right) B_{k_{1}}\left(x_{1}\right) \cdots B_{k_{m}}\left(x_{m}\right) \\
& =(-1)^{m-1} m\left(\begin{array}{l}
n \\
m
\end{array}\right) \sum_{j=0}^{m-1}(-1)^{j}\left\{\sum_{k=0}^{j}\left(\begin{array}{c}
m-j-1+k \\
k
\end{array}\right) s(m, m-j+k) y^{k}\right\} \\
& \quad \times \frac{B_{n-j}(y)}{n-j} .
\end{aligned}
$$

Theorem 3.1 can also be used to give the closed formula of sums of products of any number of Apostol-Bernoulli numbers described in [24]. For example, since the Apostol-Bernoulli 
polynomials obey the symmetric distribution

$$
\lambda \mathcal{B}_{n}(1-x ; \lambda)=(-1)^{n} \mathcal{B}_{n}\left(x ; \frac{1}{\lambda}\right) \quad(n \geq 0),
$$

and the difference equation

$$
\lambda \mathcal{B}_{n}(1+x ; \lambda)-\mathcal{B}_{n}(x ; \lambda)=n x^{n-1} \quad(n \geq 0),
$$

which can be found in [25], by setting $x_{1}=x_{2}=\cdots=x_{m}=1$ and replacing $\lambda$ by $1 / \lambda$ in Theorem 3.1, in view of (3.12), we derive

$$
\begin{gathered}
(-1)^{n} \lambda^{m} \sum_{\substack{k_{1}+\cdots+k_{m}=n \\
k_{1}, \ldots, k_{m} \geq 0}}\left(\begin{array}{c}
n \\
k_{1}, \ldots, k_{m}
\end{array}\right) \mathcal{B}_{k_{1}}(\lambda) \cdots \mathcal{B}_{k_{m}}(\lambda) \\
=\frac{(-1)^{m-1} n !}{(n-m) !} \sum_{j=0}^{m-1} p_{m, m-j-1}(m) \frac{\mathcal{B}_{n-j}\left(m ; \frac{1}{\lambda}\right)}{n-j} .
\end{gathered}
$$

On multiplying $\lambda^{x-1}$ in both sides of (3.13) and substituting $x=1, \ldots, m-1$, in view of adding the preceding results, we get for positive integer $m$ and non-negative integer $n$,

$$
\lambda^{m-1} \mathcal{B}_{n}(m ; \lambda)=\mathcal{B}_{n}(1 ; \lambda)+n \sum_{k=0}^{m-1} k^{n-1} \lambda^{k-1}
$$

We notice that, from (3.13), we have $\lambda \mathcal{B}_{1}(1 ; \lambda)=1+\mathcal{B}_{1}(\lambda)$ and $\lambda \mathcal{B}_{n}(1 ; \lambda)=\mathcal{B}_{n}(\lambda)$ for positive integer $n \geq 2$, and the following relation (see, e.g., [23]):

$$
\sum_{k=0}^{n-1} p_{n, k}(n) m^{k}=0 \quad(m, n \geq 1 \text { and } 1 \leq m \leq n-1) .
$$

Thus, by applying (3.15) and (3.16) to (3.14), one can obtain the closed formula for the Apostol-Bernoulli numbers due to Kim and $\mathrm{Hu}$ [24, Theorem 1.2], as follows:

$$
\begin{aligned}
& \sum_{\substack{k_{1}+\cdots+k_{m}=n \\
k_{1}, \ldots, k_{m} \geq 0}}\left(\begin{array}{c}
n \\
k_{1}, \ldots, k_{m}
\end{array}\right) \mathcal{B}_{k_{1}}(\lambda) \cdots \mathcal{B}_{k_{m}}(\lambda) \\
& = \begin{cases}\frac{(-1)^{m+n+1} n !}{(n-m) !} \sum_{j=0}^{m-1} p_{m, m-j-1}(m) \frac{\mathcal{B}_{n-j}\left(\frac{1}{\lambda}\right)}{n-j} & \text { if } n>m, \\
n ! p_{n, 0} \mathcal{B}_{1}(\lambda)-n ! \sum_{j=0}^{n-2} p_{n, n-j-1}(n) \frac{\mathcal{B}_{n-j}\left(\frac{1}{\lambda}\right)}{n-j} & \text { if } n=m .\end{cases}
\end{aligned}
$$

If taking $r=0$ in (3.5) then by (1.5) we obtain

$$
\begin{aligned}
& {\left[\frac{t^{n}}{n !}\right]\left(\frac{2^{m} e^{y t}}{\left(\lambda e^{t}+1\right)^{m}}\right)} \\
& \quad=\sum_{\substack{k_{1}+\cdots+k_{m}=n \\
k_{1}, \ldots, k_{m} \geq 0}}\left(\begin{array}{c}
n \\
k_{1}, \ldots, k_{m}
\end{array}\right) \mathcal{E}_{k_{1}}\left(x_{1} ; \lambda\right) \cdots \mathcal{E}_{k_{m}}\left(x_{m} ; \lambda\right) .
\end{aligned}
$$


With the help of (2.14) and (2.18), we rewrite the left-hand side of (3.18) as

$$
\begin{aligned}
& {\left[\begin{array}{c}
t^{n} \\
n !
\end{array}\right]\left(\frac{2^{m} e^{y t}}{\left(\lambda e^{t}+1\right)^{m}}\right)} \\
& \quad=2^{m-1} \sum_{k=1}^{m} \frac{(-1)^{m-k} s(m, k)}{(m-1) !}\left[\frac{t^{n}}{n !}\right]\left(e^{y t}\left(\frac{2}{\lambda e^{t}+1}\right)^{(k-1)}\right) \\
& =2^{m-1} \sum_{k=1}^{m} \frac{(-1)^{m-k} s(m, k)}{(m-1) !} \sum_{j=0}^{k-1}\left(\begin{array}{c}
k-1 \\
j
\end{array}\right)(-y)^{k-1-j} \mathcal{E}_{n+j}(y ; \lambda) .
\end{aligned}
$$

Changing the order of the summation on the right-hand side of (3.19) arises

$$
\begin{aligned}
& {\left[\begin{array}{c}
t^{n} \\
n !
\end{array}\right]\left(\frac{2^{m} e^{y t}}{\left(\lambda e^{t}+1\right)^{m}}\right)} \\
& \quad=\frac{2^{m-1}}{(m-1) !} \sum_{j=0}^{m-1}(-1)^{m-j-1} \mathcal{E}_{n+j}(y ; \lambda) \sum_{k=j+1}^{m}\left(\begin{array}{c}
k-1 \\
j
\end{array}\right) s(m, k) y^{k-1-j}
\end{aligned}
$$

The following result follows from (3.3), (3.18) and (3.20).

Theorem 3.2 Let $m$ be a positive integer and $y=x_{1}+x_{2}+\cdots+x_{m}$. Then, for positive integer $n \geq m$,

$$
\sum_{\substack{k_{1}+\cdots+k_{m}=n \\
k_{1}, \ldots, k_{m} \geq 0}}\left(\begin{array}{c}
n \\
k_{1}, \ldots, k_{m}
\end{array}\right) \mathcal{E}_{k_{1}}\left(x_{1} ; \lambda\right) \cdots \mathcal{E}_{k_{m}}\left(x_{m} ; \lambda\right)=2^{m-1} \sum_{j=0}^{m-1} p_{m, j}(y) \mathcal{E}_{n+j}(y ; \lambda) .
$$

It is worthy noticing that since (3.20) can be rewritten as

$$
\begin{aligned}
& {\left[\begin{array}{c}
t^{n} \\
n !
\end{array}\right]\left(\frac{2^{m} e^{y t}}{\left(\lambda e^{t}+1\right)^{m}}\right)} \\
& =\frac{2^{m-1}}{(m-1) !} \sum_{j=0}^{m-1}(-1)^{j} \mathcal{E}_{n+m-1-j}(y ; \lambda) \sum_{k=m-j}^{m}\left(\begin{array}{c}
k-1 \\
m-1-j
\end{array}\right) s(m, k) y^{k-(m-j)} \\
& =\frac{2^{m-1}}{(m-1) !} \sum_{j=0}^{m-1}(-1)^{j} \mathcal{E}_{n+m-1-j}(y ; \lambda) \\
& \quad \times \sum_{k=0}^{j}\left(\begin{array}{c}
m+k-j-1 \\
k
\end{array}\right) s(m, m+k-j) y^{k},
\end{aligned}
$$

by combining (3.18) and (3.22), we obtain

$$
\begin{aligned}
& \sum_{\substack{k_{1}+\cdots+k_{m}=n \\
k_{1}, \ldots, k_{m} \geq 0}}\left(\begin{array}{c}
n \\
k_{1}, \ldots, k_{m}
\end{array}\right) \mathcal{E}_{k_{1}}\left(x_{1} ; \lambda\right) \cdots \mathcal{E}_{k_{m}}\left(x_{m} ; \lambda\right) \\
& =\frac{2^{m-1}}{(m-1) !} \sum_{j=0}^{m-1}(-1)^{j}\left\{\sum_{k=0}^{j}\left(\begin{array}{c}
m+k-j-1 \\
k
\end{array}\right) s(m, m+k-j) y^{k}\right\} \mathcal{E}_{n+m-1-j}(y ; \lambda) .
\end{aligned}
$$


Obviously the case $\lambda=1$ in (3.23) gives the formula of Dilcher [19, Theorem 5] on the classical Euler polynomials.

We next consider the case $1 \leq r \leq m-1$ in (3.5). For convenience, in the following we always denote $z=\lambda e^{t}$. We apply the familiar partial fraction decomposition and let

$$
\frac{1}{(z-1)^{r}(z+1)^{m-r}}=\sum_{i=0}^{r-1} \frac{M_{i}}{(z-1)^{r-i}}+\sum_{j=0}^{m-r-1} \frac{N_{j}}{(z+1)^{m-r-j}} .
$$

We are now in the position to determine the coefficients $M_{i}$ and $N_{j}$ in (3.24). By multiplying both sides in (3.24) by $(z-1)^{r}$ and taking $z \rightarrow 1$, we obtain

$$
M_{0}=\lim _{z \rightarrow 1} \frac{1}{(z+1)^{m-r}}=\frac{1}{2^{m-r}} .
$$

For the case $1 \leq i \leq r-1$ in (3.24), by multiplying $(z-1)^{r-i}$ in both sides of (3.24) and taking $z \rightarrow 1$, with the help of the binomial theorem $(z+1)^{n}=\sum_{k=0}^{n}\left(\begin{array}{l}n \\ k\end{array}\right)(z-1)^{k} 2^{n-k}$ for non-negative integer $n$, we get

$$
\begin{aligned}
M_{i} & =\lim _{z \rightarrow 1}(z-1)^{r-i}\left\{\frac{1}{(z-1)^{r}(z+1)^{m-r}}-\sum_{k=0}^{i-1} \frac{M_{k}}{(z-1)^{r-k}}\right\} \\
& =\frac{1}{2^{m-r}} \lim _{z \rightarrow 1} \frac{1-(z+1)^{m-r} \sum_{k=0}^{i-1} M_{k}(z-1)^{k}}{(z-1)^{i}} \\
& =\frac{1}{2^{m-r}} \lim _{z \rightarrow 1} \frac{1-\sum_{j=0}^{m-r} \sum_{k=0}^{i-1}\left(\begin{array}{c}
m-r \\
j
\end{array}\right) 2^{m-r-j} M_{k}(z-1)^{k+j}}{(z-1)^{i}} .
\end{aligned}
$$

In light of (3.25), we can make the L'Hôspital rule for (3.26). In fact, repeatedly applying the L'Hôspital rule $i$ times there arises

$$
M_{i}=-\frac{1}{i !} \lim _{z \rightarrow 1} \sum_{j=0}^{m-r} \sum_{k=0}^{i-1}\left(\begin{array}{c}
m-r \\
j
\end{array}\right) \frac{M_{k}(k+j)_{i}}{2^{j}}(z-1)^{k+j-i}
$$

where $(x)_{n}$ is the falling factorial $(x)_{n}$ of order $n$ in (2.1). Hence, the coefficients $M_{i}$ satisfy

$$
M_{0}=\frac{1}{2^{m-r}} \quad \text { and } \quad M_{i}=-\sum_{k=0}^{i-1}\left(\begin{array}{c}
m-r \\
i-k
\end{array}\right) \frac{M_{k}}{2^{i-k}} \quad(1 \leq i \leq r-1) .
$$

It follows from (3.28) that we can claim

$$
M_{i}=\frac{(-1)^{i}}{2^{m-r+i}}\left(\begin{array}{c}
m-r+i-1 \\
i
\end{array}\right) \quad(0 \leq i \leq r-1)
$$

We shall use induction on $i$. Obviously (3.29) holds trivially when $i=0$. Assume that (3.29) is complete for any smaller value of $i$. From (3.28) and (3.29), we have

$$
M_{i}=-\frac{1}{2^{m-r+i}} \sum_{k=0}^{i}\left(\begin{array}{c}
m-r \\
i-k
\end{array}\right)\left(\begin{array}{c}
m-r+k-1 \\
k
\end{array}\right)(-1)^{k}+\frac{(-1)^{i}}{2^{m-r+i}}\left(\begin{array}{c}
m-r+i-1 \\
i
\end{array}\right) .
$$


Note that, for non-negative integer $n$ and complex numbers $a$ and $b$ ( $c f$. the ChuVandermonde summation formula stated in [45]),

$$
\sum_{k=0}^{n} \frac{\langle-n\rangle_{k} \cdot\langle a\rangle_{k}}{k ! \cdot\langle b\rangle_{k}}=\frac{\langle b-a\rangle_{n}}{\langle b\rangle_{n}}
$$

and the first summation of the right-hand side of (3.30) can be rewritten as

$$
\begin{aligned}
& \sum_{k=0}^{i}\left(\begin{array}{c}
m-r \\
i-k
\end{array}\right)\left(\begin{array}{c}
m-r+k-1 \\
k
\end{array}\right)(-1)^{k} \\
& \quad=\frac{m-r}{i !} \sum_{k=0}^{i} \frac{(-i)(-i-1) \cdots(-i+k-1) \cdot(m-r+k-1) !}{k ! \cdot(m-r+k-i) !} \\
& \quad=\frac{m-r}{i !} \cdot \frac{(m-r-1) !}{(m-r-i) !} \sum_{k=0}^{i} \frac{\langle-i\rangle_{k} \cdot\langle m-r\rangle_{k}}{k ! \cdot\langle m-r-i+1\rangle_{k}},
\end{aligned}
$$

where $\langle x\rangle_{n}$ is the rising factorial $\langle x\rangle_{n}$ of order $n$ in Theorem 2.2. It follows from (3.31) and (3.32) that

$$
\sum_{k=0}^{i}\left(\begin{array}{c}
m-r \\
i-k
\end{array}\right)\left(\begin{array}{c}
m-r+k-1 \\
k
\end{array}\right)(-1)^{k}=0
$$

which together with (3.30) immediately yields (3.29). On multiplying both sides in (3.24) by $(z+1)^{m-r}$ and then taking $z \rightarrow-1$, we obtain

$$
N_{0}=\lim _{z \rightarrow-1} \frac{1}{(z-1)^{r}}=\frac{1}{(-2)^{r}} \text {. }
$$

On multiplying $(z+1)^{m-r-j}$ in both sides of (3.24) and then taking $z \rightarrow-1$, with the help of the binomial theorem, we get, for $1 \leq j \leq m-r-1$,

$$
\begin{aligned}
N_{j} & =\lim _{z \rightarrow-1}(z+1)^{m-r-j}\left\{\frac{1}{(z-1)^{r}(z+1)^{m-r}}-\sum_{k=0}^{j-1} \frac{N_{k}}{(z+1)^{m-r-k}}\right\} \\
& =\frac{1}{(-2)^{r}} \lim _{z \rightarrow-1} \frac{1-(z-1)^{r} \sum_{k=0}^{j-1} N_{k}(z+1)^{k}}{(z+1)^{j}} \\
& =\frac{1}{(-2)^{r}} \lim _{z \rightarrow-1} \frac{1-\sum_{i=0}^{r} \sum_{k=0}^{j-1}\left(\begin{array}{l}
r \\
i
\end{array}\right)(-2)^{r-i} N_{k}(z+1)^{k+i}}{(z+1)^{j}} .
\end{aligned}
$$

So from (3.34), we repeatedly apply the L'Hôspital rule $j$ times to get

$$
N_{0}=\frac{1}{(-2)^{r}} \quad \text { and } \quad N_{j}=-\sum_{k=0}^{j-1}\left(\begin{array}{c}
r \\
j-k
\end{array}\right) \frac{N_{k}}{(-2)^{j-k}} \quad(1 \leq j \leq m-r-1) \text {. }
$$

By a similar consideration to (3.29), we can state

$$
N_{j}=\frac{(-1)^{j}}{(-2)^{r+j}}\left(\begin{array}{c}
r+j-1 \\
j
\end{array}\right) \quad(0 \leq j \leq m-r-1) .
$$


It follows from (3.5), (3.24), (3.29) and (3.37) that, for positive integers $m$ and $r$ with $1 \leq$ $r \leq m-1$,

$$
\begin{aligned}
& \sum_{\substack{k_{1}+\cdots+k_{m}=n \\
k_{1}, \ldots, k_{m} \geq 0}}\left(\begin{array}{c}
n \\
k_{1}, \ldots, k_{m}
\end{array}\right) \mathcal{B}_{k_{1}}\left(x_{1} ; \lambda\right) \cdots \mathcal{B}_{k_{r}}\left(x_{r} ; \lambda\right) \mathcal{E}_{k_{r+1}}\left(x_{r+1} ; \lambda\right) \cdots \mathcal{E}_{k_{m}}\left(x_{m} ; \lambda\right) \\
& =\sum_{i=0}^{r-1}\left(\begin{array}{c}
m-r+i-1 \\
i
\end{array}\right) \frac{(-1)^{i} n !}{2^{i}(n-i) !}\left[\frac{t^{n-i}}{(n-i) !}\right]\left(\frac{t^{r-i} e^{y t}}{\left(\lambda e^{t}-1\right)^{r-i}}\right) \\
& \quad+(-1)^{r} 2^{-r} \sum_{j=0}^{m-r-1}\left(\begin{array}{c}
r+j-1 \\
j
\end{array}\right) \frac{n !}{(n-r) !} \\
& \quad \times\left[\frac{t^{n-r}}{(n-r) !}\right]\left(\frac{2^{m-r-j} e^{y t}}{\left(\lambda e^{t}+1\right)^{m-r-j}}\right) .
\end{aligned}
$$

Thus, applying (3.9) and (3.20) to (3.38), in light of (3.3), we get the result.

Theorem 3.3 Let $m$ be a positive integer and $y=x_{1}+x_{2}+\cdots+x_{m}$. Then, for positive integer $n \geq m$ and positive integer $r$ with $1 \leq r \leq m-1$,

$$
\begin{aligned}
& \sum_{\substack{k_{1}+\cdots+k_{m}=n \\
k_{1}, \ldots, k_{m} \geq 0}}\left(\begin{array}{c}
n \\
k_{1}, \ldots, k_{m}
\end{array}\right) \mathcal{B}_{k_{1}}\left(x_{1} ; \lambda\right) \cdots \mathcal{B}_{k_{r}}\left(x_{r} ; \lambda\right) \mathcal{E}_{k_{r+1}}\left(x_{r+1} ; \lambda\right) \cdots \mathcal{E}_{k_{m}}\left(x_{m} ; \lambda\right) \\
& =\frac{(-1)^{r-1} n !}{(n-r) !} \sum_{i=0}^{r-1}\left(\begin{array}{c}
m-r+i-1 \\
i
\end{array}\right) \frac{1}{2^{i}} \sum_{k=0}^{r-1-i} p_{r-i, r-i-k-1}(y) \frac{\mathcal{B}_{n-i-k}(y ; \lambda)}{n-i-k} \\
& \quad+\frac{(-1)^{r} n !}{2^{r}(n-r) !} \sum_{j=0}^{m-r-1}\left(\begin{array}{c}
r+j-1 \\
j
\end{array}\right) 2^{m-r-1-j} \\
& \quad \times \sum_{k=0}^{m-r-1-j} p_{m-r-j, k}(y) \mathcal{E}_{n-r+k}(y ; \lambda) .
\end{aligned}
$$

\section{Competing interests}

The authors declare that they have no competing interests.

\section{Authors' contributions}

All authors contributed equally to the writing of this paper. All authors read and approved the final manuscript.

\section{Author details}

${ }^{1}$ Faculty of Science, Kunming University of Science and Technology, Kunming, Yunnan 650500, People's Republic of

China. ${ }^{2}$ Faculty of Economics, Administrative and Social Sciences, Hasan Kalyoncu University, Gaziantep, 27410, Turkey.

\section{Acknowledgements}

This work is supported by the Foundation for Fostering Talents in Kunming University of Science and Technology (Grant No. KKSY201307047) and the National Natural Science Foundation of P.R. China (Grant Nos. 11326050, 11071194).

\section{Received: 21 March 2014 Accepted: 15 May 2014 Published: 27 May 2014}

\section{References}

1. Cohen, H: Number Theory, Volume II: Analytic and Modern Tools. Graduate Texts in Math., vol. 240. Springer, New York (2007)

2. Nielsen, N: Traité élémentaire des nombres de Bernoulli. Gauthier-Villars, Paris (1923)

3. Nörlund, NE: Vorlesungen über Differenzenrechnung. Springer, Berlin (1924)

4. Srivastava, HM, Choi, J: Zeta and q-Zeta Functions and Associated Series and Integrals. Elsevier, Amsterdam (2012) 
5. Nörlund, NE: Mémoire sur les polynomes de Bernoulli. Acta Math. 43, 121-196 (1922)

6. He, Y, Zhang, WP: Some sum relations involving Bernoulli and Euler polynomials. Integral Transforms Spec. Funct. 22, 207-215 (2011)

7. He, Y, Wang, CP: Some formulae of products of the Apostol-Bernoulli and Apostol-Euler polynomials. Discrete Dyn. Nat. Soc. 2012, Article ID 927953 (2012)

8. He, Y, Wang, CP: Recurrence formulae for Apostol-Bernoulli and Apostol-Euler polynomials. Adv. Differ. Equ. 2012, Article ID 209 (2012)

9. He, Y, Zhang, WP: A convolution formula for Bernoulli polynomials. Ars Comb. 108, 97-104 (2013)

10. He, Y, Zhang, WP: A three-term reciprocity formula for Bernoulli polynomials. Util. Math. (to appear)

11. Wang, JZ: New recurrence formulae for the Apostol-Bernoulli and Apostol-Euler polynomials. Adv. Differ. Equ. 2013, Article ID 247 (2013)

12. Carlitz, L: Note on the integral of the product of several Bernoulli polynomials. J. Lond. Math. Soc. 34, 361-363 (1959)

13. Agoh, T, Dilcher, K: Convolution identities and lacunary recurrences for Bernoulli numbers. J. Number Theory 124 , 105-122 (2007)

14. Agoh, T, Dilcher, K: Reciprocity relations for Bernoulli numbers. Am. Math. Mon. 115, 237-244 (2008)

15. Eie, M: A note on Bernoulli numbers and Shintani generalized Bernoulli polynomials. Trans. Am. Math. Soc. 348 1117-1136 (1996)

16. Sitaramachandrarao, R, Davis, B: Some identities involving the Riemann zeta function, II. Indian J. Pure Appl. Math. 17, 1175-1186 (1986)

17. Sankaranarayanan, A: An identity involving Riemann zeta function. Indian J. Pure Appl. Math. 18, 794-800 (1987)

18. Zhang, WP: On the several identities of Riemann zeta-function. Chin. Sci. Bull. 36, 1852-1856 (1991)

19. Dilcher, K: Sums of products of Bernoulli numbers. J. Number Theory 60, 23-41 (1996)

20. Petojević, A: A note about the sums of products of Bernoulli numbers. Novi Sad J. Math. 37, 123-128 (2007)

21. Petojević, A: New sums of products of Bernoulli numbers. Integral Transforms Spec. Funct. 19, 105-114 (2008)

22. Petojević, A, Srivastava, HM: Computation of Euler's type sums of the products of Bernoulli numbers. Appl. Math. Lett. 22, 796-801 (2009)

23. Kim, M-S: A note on sums of products of Bernoulli numbers. Appl. Math. Lett. 24, 55-61 (2011)

24. Kim, M-S, Hu, S: Sums of products of Apostol-Bernoulli numbers. Ramanujan J. 28, 113-123 (2012)

25. Luo, QM, Srivastava, HM: Some generalizations of the Apostol-Bernoulli and Apostol-Euler polynomials. J. Math. Anal. Appl. 308, 290-302 (2005)

26. Luo, QM: Apostol-Euler polynomials of higher order and Gaussian hypergeometric functions. Taiwan. J. Math. 10, 917-925 (2006)

27. Apostol, TM: On the Lerch zeta function. Pac. J. Math. 1, 161-167 (1951)

28. Srivastava, HM: Some formulas for the Bernoulli and Euler polynomials at rational arguments. Math. Proc. Camb. Philos. Soc. 129, 77-84 (2000)

29. Choi, J, Jang, DS, Srivastava, HM: A generalization of the Hurwitz-Lerch zeta function. Integral Transforms Spec. Funct. 19,65-79 (2008)

30. Garg, M, Jain, K, Srivastava, HM: Some relationships between the generalized Apostol-Bernoulli polynomials and Hurwitz-Lerch zeta functions. Integral Transforms Spec. Funct. 17, 803-815 (2006)

31. Lu, D-Q, Srivastava, HM: Some series identities involving the generalized Apostol type and related polynomials. Comput. Math. Appl. 62, 3591-3602 (2011)

32. Luo, QM, Srivastava, HM: Some relationships between the Apostol-Bernoulli and Apostol-Euler polynomials. Comput. Math. Appl. 51, 631-642 (2006)

33. Luo, QM: Fourier expansions and integral representations for the Apostol-Bernoulli and Apostol-Euler polynomials. Math. Comput. 78, 2193-2208 (2009)

34. Luo, QM: The multiplication formulas for the Apostol-Bernoulli and Apostol-Euler polynomials of higher order. Integral Transforms Spec. Funct. 20, 377-391 (2009)

35. Navas, LM, Ruiz, FJ, Varona, JL: Asymptotic estimates for Apostol-Bernoulli and Apostol-Euler polynomials. Math. Comput. 81, 1707-1722 (2011)

36. Prévost, M: Padé approximation and Apostol-Bernoulli and Apostol-Euler polynomials. J. Comput. Appl. Math. 233, 3005-3017 (2010)

37. Srivastava, HM, Todorov, PG: An explicit formula for the generalized Bernoulli polynomials. J. Math. Anal. Appl. 130, 509-513 (1988)

38. Srivastava, HM, Garg, M, Choudhary, S: A new generalization of the Bernoulli and related polynomials. Russ. J. Math. Phys. 17, 251-261 (2010)

39. Xu, A-M, Cen, Z-D: Some identities involving exponential functions and Stirling numbers and applications. J. Comput. Appl. Math. 260, 201-207 (2014)

40. Guo, B-N, Qi, F: Some identities and an explicit formula for Bernoulli and Stirling numbers. J. Comput. Appl. Math. 255 568-579 (2014)

41. Comtet, L: Advanced Combinatorics: The Art of Finite and Infinite Expansions. Reidel, Dordrecht (1974)

42. Koumandos, S: On Ruijsenaars' asymptotic expansion of the logarithm of the double gamma function. J. Math. Anal. Appl. 341, 1125-1132 (2008)

43. Qi, F: Eight interesting identities involving the exponential function, derivatives, and Stirling numbers of the second kind. arXiv:1202.2006

44. He, Y, Zhang, WP: Some symmetric identities involving a sequence of polynomials. Electron. J. Comb. 17, Article ID N7 (2010)

45. Gasper, G, Rahman, M: Basic Hypergeometric Series. Cambridge University Press, Cambridge (1990)

10.1186/1687-1847-2014-155

Cite this article as: He and Araci: Sums of products of Apostol-Bernoulli and Apostol-Euler polynomials. Advances in Difference Equations 2014, 2014:155 